\title{
Intelligent Systems Technologies for Ops
}

\author{
Ernest E. Smith ${ }^{1}$ and David J. Korsmeyer ${ }^{2}$ \\ NASA Ames Research Center, Moffett Field, Ca, 94035, USA
}

\begin{abstract}
As NASA supports International Space Station assembly-complete operations through 2020 (or later) and prepares for future human exploration programs, there is additional emphasis in the manned spaceflight program to find more efficient and effective ways of providing the ground-based mission support. Since 2006 this search for improvement has led to a significant cross-fertilization between the NASA advanced software development community and the manned spaceflight operations community. A variety of mission operations systems and tools have been developed over the past decades as NASA has operated the Mars robotic missions, the Space Shuttle, and the International Space Station. NASA Ames Research Center has been developing and applying its advanced intelligent systems research to mission operations tools for both unmanned Mars missions operations since 2001 and to manned operations with NASA Johnson Space Center since 2006. In particular, the fundamental advanced software development work under the Exploration Technology Program, and the experience and capabilities developed for mission operations systems for the Mars surface missions (Spirit/Opportunity, Phoenix Lander, and MSL) have enhanced the development and application of advanced mission operation systems for the International Space Station and future spacecraft. This paper provides an update on the status of the development and deployment of a variety of intelligent systems technologies adopted for manned mission operations and some discussion of the planned work for Autonomous Mission Operations in future human exploration. We discuss several specific projects between the Ames Research Center and the Johnson Space Center's Mission Operations Directorate, and how these technologies and projects are enhancing the mission operations support for the International Space Station, and supporting the current Autonomous Mission Operations Project for the mission operation support of the future human exploration programs.
\end{abstract}

\section{Introduction}

The National Aeronautics and Space Administration (NASA) is posturing itself for the next major step in the exploration of space, and will require the development of many new capabilities including the design of new spacecraft, new launch services, and new processes and tools associated with the mission operations support. The mission operations support includes the planning of the NASA missions, the training of the crew and flight control team, and the mission execution. The specific targets for NASA mission operations beyond the International Space Station (ISS) Program are currently being assessed by the Human Exploration Framework Team (HEFT) and it is clear that NASA will need to infuse new technologies into the new space exploration initiatives. The range and complexity of these exploration missions will require an unprecedented use of automation and robotics in support of human crews. This will require the operations of manned spacecraft in close conjunction with planetary robotic systems.

NASA's current space flight missions are still largely segmented into unmanned missions funded by the NASA's Science Mission Directorate and the human spaceflight missions operated by the Human Exploration and Operations Mission Directorate. Typically the organizations within NASA that operate the unmanned missions are different from the organizations that operated crewed space systems. The mission operations requirements and needs for the

\footnotetext{
${ }^{1}$ Project Integration Manager, Intelligent Systems Division, detailed at JSC, Mail Code DD.

${ }^{2}$ Chief, Intelligent Systems Division, Mail Stop 269-1, AIAA Fellow.
} 
robotic missions have been relatively distinct from those for the Space Shuttle and the International Space Station (ISS).

Developing and validating any new exploration spacecraft and its associated infrastructure places requirements on operations design for future exploration missions - as the development of systems during the Constellation Program has shown. Separate mission operations processes - and cultures - have evolved to support manned missions and unmanned/robotic missions, each appropriately geared to the unique challenges of the two classes of missions. ${ }^{1}$ Developing and maturing the advanced software technologies and process innovations that can benefit both the robotic missions and to also benefit crewed missions is not simple or straightforward. NASA Ames Research Center has been working with both human spaceflight and robotic systems communities now for several years developing mission operations tools and system and is helping to bridge the gap between tools for manned and robotic mission operations.

\section{A. Mission Operations Directorate Needs}

In collaboration with the NASA's Astronaut corps, the Johnson Space Center's (JSC) Mission Operations Directorate (MOD) manages and maintains the flight operations of all of NASA's human spaceflights. These include all of the previous manned spaceflights from Mercury through Apollo, and now the Space Shuttle and ISS. The flight operations of the crewed elements of the future exploration initiatives will likely also be managed by MOD, though MOD's involvement in the potential commercial human spaceflight operations is still evolving.

Manned flight operations support is provided by the combination of several ground mission control centers around the U.S. and the World, primarily focused through the Mission Control Center (MCC) at Johnson Space Center (JSC) in Houston, Texas. The ISS is supported by both the MCC and the Payload Ops Integration Center (POIC) at Marshal Space Flight Center (MSFC) in Huntsville, Alabama, plus the International Partners control centers including the Mission Control Center in Moscow (MCC-M or TsUP), the European Space Agency (ESA) Columbus Control Center in Germany and the ATV Control Center in France, the Canadian Space Agency (CSA) Control Center in St. Hubert, Canada, and the Japanese Experiment Module (JEM) and HTV Control Centers in Tsukuba, Japan.

With the retirement of the Shuttle in 2011, MOD at JSC is preparing for future exploration initiatives and is achieving the stated a goal of reducing the manpower required to support manned space flight operations. MOD has restructured itself to be more efficient in support of the "Plan, Train, and Fly" activities associated with manned flight operations and is also investing in several technology infusion opportunities as described within this paper which they anticipate will help in achieving this reduction goal.

\section{Intelligent Systems at NASA}

\section{A. Advanced Software Systems for Operations}

NASA Ames Research Center has long been a leader in the development of advanced software technologies and systems for NASA Missions. Through the 1990s this role included leadership of the majority of NASA's automated reasoning and human-centered computing programs. In the first decade of this millennium, Ames has lead all of the advanced software research and development projects for the Exploration Systems Mission Directorate. Ames has exercised these responsibilities to provide the Agency with a notable set of software technology "firsts," including the first autonomy software to be flown by NASA on a spacecraft ${ }^{2}$ and the first advanced planning software to daily plan a robotic planetary mission. ${ }^{3}$ Similarly the multiyear partnership with the manned spaceflight operations described in this paper has grown out of a combination of excellent technical work, a focus on NASA's needs and vision of its future, and a portfolio of activities ranging from needs-driven technology development to projectfocused tool development.

The partnership between NASA Ames Intelligent Systems and NASA JSC's MOD has been addressing the significant challenges posed by the agency's long-term operations of manned spacecraft. The knowledge, intelligence, and engineering analysis currently provided by MOD mission controllers will need to be automated and accompany the astronauts on future missions to both the ISS and exploration destinations. As ISS, the National Laboratory, becomes a major test-bed for systems, mission operations will need to be more adaptable for varied mission scenarios. An ability to rapidly and dependably develop and modify software could provide MOD the means to alter system capabilities on the fly. Following current practices, software modifications to space-based and flight control systems can take in the months or years to make. To modify capabilities between and during missions, revolutionary software development approaches are needed - new approaches that, in the tens of minutes, can result in effective and dependable modifications. Like MOD, in order to achieve these goals Ames must target an 
evolutionary path to proving out technical approaches. During this evolution it must validate the value for the crewed mission operations community.

\section{B. Human-Centered Computing}

The lessons learned from NASA Ames developers and the experience they had creating tools for the Jet Propulsion Laboratory's Mars planetary missions and the past six years of collaboration with MOD have defined a framework for how Ames and JSC determine what opportunities exist for intelligent systems applied to the crewed spacecraft operations for NASA. Principally the discussion of new technologies and tools needs to be framed in a manner that identifies and emphasizes the value to the MOD operational flight controller. It is not about replacing 'man with a machine', but about complementing and augmenting the flight controller or operator to do a better job. This overall methodology and approach is often referred to as human-centered computing.

Human-centered computing looks to the processes and procedures that people do to perform any given job, then with these understanding attempts to identify opportunities to improve these processes and procedures. In particular, for mission operations, improvements are quantified by specifically identifying how a tool can increase a person's efficiency, enhance a person's functional capability, and/or improve the assurance of a person's decisions.

A human-centered computing strategy contains the following essential elements:

- Deploy personnel to mission centers to work with the NASA customer(s) to understand the exact nature of their current work and future challenges that may be amenable to software solutions.

- Scout for all relevant approaches or technologies that may address the customer's needs.

- Identify technology gaps left by current software capabilities to seed new research and development.

- Simultaneously conduct carefully-targeted research and development to address the gaps on an ongoing basis.

- Evaluate and compare competing results, working closely with the customer to determine the strengths and weaknesses and the cost-benefit of the each candidate solution and improve it on this basis.

This above strategy worked exceptionally well for infusing advanced technologies in the Mars robotic missions with Spirit and Opportunity rover, the Phoenix Lander, and the upcoming Mars Science Laboratory. It has proven to work equally well for the human spaceflight community operating Space Station and the Shuttle.

\section{Advanced Mission Operations Development Projects}

At the advent of the Constellation Program, Ames and JSC began discussions about how to leverage each other's strengths and capabilities, and established a set of initial projects that addressed outstanding needs within MOD. These projects first started in 2006 and have progressed to several full-scale projects incrementally delivering operational flight controller tools planned through 2014. All of these mission operations development projects are considered relevant to increasing the efficiency of the mission operations flight control team, and significantly support the goal of overall operations manpower reductions while ensuring the high level of safety for mission operations. Even with the cancellation of the Constellation Program, the need for innovative and efficient mission operations remains. The HEFT studies have pointed to Crew-centered Operations at remote destination of NearEarth Objects (NEOs) and asteroids as the desired operation target. Additionally the continuation of ISS operations until at least 2020 means that operational efficiency is a driving factor in keeping costs of the ISS flying minimum. A synopsis status of some of the projects is presented below.

\section{A. Mission Planning System Enhancements}

The On-Board Short Term Plan Viewer (OSTPV) is a tool used by flight controllers to view and manipulate the International Space Station's Short Term Plan (STP). This plan spans several days to 2 weeks, and describes activities performed by ISS crew and the status of major ISS subsystems at a time granularity of tens of minutes. These plans are developed using the Consolidated Planning System (CPS), which contains rules that govern the legality of the STP. Flight controllers can manipulate the STP by re-scheduling or deleting activities; however, OSTPV only displays the new schedule, and does not perform any checks for constraints that may be violated as a consequence of plan manipulations.

The MOD Operations Planning Timeline Integration System (OPTimIS - previously referred to as the Next Generation Planning System (NGPS)) project will develop one planning system and process for all programs to leverage with technologies to improve and simplify mission planning for all MOD teams. The project will incorporate enhanced scheduling capabilities based on the Ames Scheduling and Planning Interface for Exploration (SPIFe) tools and will utilize the Ames Extensible Universal Remote Operations Planning Architecture (EUROPA) tools to check for constraints violations. The collaboration between MOD, their contractors, and the Ames technologists will 
consolidate many of the disparate planning tools being used today. ${ }^{4}$ This is envisioned to have a significant impact on the overall efficiency of the planning efforts for ISS.

\section{B. Mission Operations Design Modeling and Workflow Automation}

OCAMS (Orbital Communication Adapter Mirroring System) is a practical engineering application of multiagent systems technology, using the Business Redesign Agent-based Holistic Modeling System (BRAHMS) modeling and simulation tool. ${ }^{5}$ The BRAHMS system combines models of systems (e.g., robots, tools, software) with models of people communicating and moving in a simulated geographic space, revealing how interactions of people, facilities, and tools are productive or gaps that may occur in capabilities and procedures. BRAHMS simulations can be converted into a runtime system in which software agents automate and mediate work flow operations and communications among people and systems.

The project began in November 2006, leading to a completed workflow automation system that is currently being used by OCA Officers in the MPSR backroom supporting the ISS. The OCA Officer is responsible for uplinking and down-linking all files to and from the ISS, based on scheduled operations and requests from ground support. The OCA Officer mirrors, archives, and forwards files to support personnel and documents this work in flight notes and logs. The Ames OCAMS project team has developed the agent-based OCAMS to perform this entire process by using the Simulation-to-Implementation Engineering method. ${ }^{6}$ First they simulated the OCA Officer's work practices to identify possible process improvements. Using statistics generated from this simulation model and collaborative design with the OCA Team at JSC, they then developed an agent-based workflow system that supports the redesigned and improved OCA work process. With the OCAMS system, the time spent by the OCA Officer processing files uplinked and down-linked to the Space Station has been sufficiently reduced to enable merging this console position, thereby reducing the manpower required to support this function.

\section{Advanced Systems Monitoring}

The Anomaly Monitoring Inductive Software System (AMISS) is an Ames developed health monitoring software application that compares current system data with data from previous nominal system operations. AMISS is based on the Ames developed Inductive Monitoring System (IMS), and applies data mining techniques to archived telemetry to establish a baseline of normal behavior for groups of data parameters from the monitored system. AMISS then uses that baseline to identify off-normal behavior in real-time telemetry, potentially alerting the mission operations team to problems prior to any caution and warning annunciation for the system. Any deviations from normal baseline behavior will be indicated by AMISS with a non-zero "distance" from nominal. Information is also provided on which data parameters are contributing to the off-nominal readings to help identify the source of the anomaly. ${ }^{7}$

In 2006, Ames delivered AMISS based tools to JSC that allow mission operations users to retrieve archived mission data and run the data offline on AMISS to both "train" the tool on nominal data and to execute the "monitoring" feature. This capability was tested on several ISS Control Moment Gyroscope (CMG) data sets, including data collected during some significant CMG malfunctions. AMISS successfully detected anomalies in CMG behavior in these data sets, sometimes several hours before malfunctions were detected by current MCC systems. These promising results prompted JSC to establish a 2007-08 task to deploy the tool within the MCC environment for evaluation and use in real time by the on-console flight control team. The AMISS tool has been integrated with the MCC real time data system and deployed on the mission control consoles in the ISS control room to provide real time CMG monitoring. In addition, AMISS has been augmented with fault detection routines that will automatically detect and identify some common CMG faults to assist controllers in diagnosis and recovery activities. AMISS has also been applied to the monitoring of the ISS External Thermal Control System (ETCS) subsystems.

\section{Mission Control Technologies}

Current MOD mission operations systems are built as a collection of monolithic software applications. Each application serves the needs of a specific user base associated with a discipline or functional role. Designed to accomplish specific tasks, each application embodies specialized functional knowledge and has its own data storage, data models, programmatic interfaces, user interfaces, and customized business logic. In effect, each application creates its own walled-off environment. While individual applications are sometimes reused across multiple missions, it is expensive and time consuming to maintain these systems, and both costly and risky to upgrade them in the light of new requirements or modify them for new purposes. It is even more expensive to achieve new integrated activities across a set of monolithic applications.

These problems impact the life-cycle cost (especially design, development, testing, training, maintenance, and integration) of each new mission operations system. They also inhibit system innovation and evolution. This in turn 
hinders NASA's ability to adopt new operations paradigms, including increasingly automated space systems, such as autonomous rovers, autonomous onboard crew systems, and integrated control of human and robotic missions.

In order to achieve NASA's vision of affordably and reliably, we need to consider and mature new ways to build mission control systems that overcome the problems inherent in systems of monolithic applications. Two keys to the solution are modularity and interoperability. Modularity will increase extensibility, reusability, and maintainability. Interoperability will enable composition of larger systems out of smaller parts, and make possible the construction of new integrated activities that tie together, at a deep level, the capabilities of many of the components. Modularity and interoperability together contribute to flexibility.

The Mission Control Technologies (MCT) Project, ${ }^{8}$ a collaboration of multiple NASA Centers led by Ames, is building a framework (based upon the open-source Eclipse software) to enable software to be assembled from flexible collections of components and services. MCT has been executing in the MCC Operations Test Facility (OTF) for several years, shadowing ISS mission operations. It has now been deployed into the MCC to expand the user base that can evaluate the cyclic deliveries of this capability and to develop, gather, and analyze measurements to evaluate the performance and usability of MCT, from a flight controller's perspective. This move also enhances our ability to define and analyze the proper engineering metrics - performance, lines of code, and the potential cost savings.

\section{E. Search Tools}

The cross search or "XSearch" project focused on improving MOD access to and retrieval of critical information required to monitor, control, and manage ISS and Space Shuttle. ${ }^{9}$ While much of this information (in the form of notes, change requests, action item lists, procedures, documentation, etc.) was accessible using a patchwork of disconnected tools and databases, this project built a unified search capability across these data sources thereby presenting a unified single Web-based interface for all MOD flight controllers. In addition, the system identifies cross-referenced and other relevant information that flight controllers might otherwise overlook.

The XSearch development, working closely with MOD Flight Controllers, began in January 2006 and has been deployed in the MCC environment enhancing the cross-database searches (initially the mission Flight Notes, Anomaly Reports and Chits) for the flight control team. In addition to search, the XSearch system provides two other important capabilities: cross-referencing and similarity detection. For the final phase of the project which ended in September 2010, XSearch incorporated the searching of MOD Flight Techniques and Joint Operations Panel meeting minutes.

\section{F. Training Management}

Scheduling, Training Administration, and Records (STAR) was developed as NASA's next-generation training management system, primarily for the ISS flight crew and to a lesser extent the Instructors, and Flight Controllers. It replaced the existing Training Administration Management System (TAMS) with a suite of tools that provide integrated curriculum development and documentation, customized training plans, scheduling of personnel and facilities, training event feedback, and other training resources. The primary return-on-investment for STAR is the reduction of the number of planners and schedulers required for MOD personnel training and facility scheduling. All modules were designed for maximum efficiency and interoperability. In an iterative process, event feedback was used to help optimize the training plans. Retirement of the oldest MOD training tool, TAMS, was accomplished following the final Shuttle mission, and reduced the overall sustaining costs for the training applications. STAR is currently in sustaining and further development is not planned at this time.

\section{G. Solar Array Management}

As the construction of the ISS was being completed, the full complement of solar arrays was added to provide the power required to support additional modules on the larger station. These new arrays had more freedom to articulate, enabling better tracking of the sun and thus increased power production. However, these arrays also had more complex constraints that limit the range of safe orientations, due to structural loads, contamination concerns, and thermal impacts. These limitations on safe array orientations impact power generation, which requires MOD flight controllers to constantly balance multiple complex constraints against ISS power needs. The increased complexity does not only impact pre-planning activities, but has an even more acute effect on real-time operations, in particular when handling unexpected events or changes in operations plans.

The Solar Array Constraint Engine (SACE) project has developed a tool that provides intelligent decisionsupport capabilities to ISS power systems flight controllers, to assist them with the task of planning and executing solar array operations in a safe and effective manner. ${ }^{10}$ SACE provides situational awareness, orientation evaluation and optimization, and array operations planning functionality to flight controllers. The SACE tool is built on the EUROPA engine, which provides constraint management and reasoning, decision-support and planning. 
The SACE tool enables simple solar array plan generation. SACE includes: (1) separation of plan configuration and generation into separate phases, so that the user can modify the configuration before proceeding with plan generation; (2) improved algorithm for consolidating constraints and user restrictions during the solution of configurations that need to be merged during plan generation; (3) visual indication to the user of configurations merged during planning; (4) reporting of orbital-average power availability for the generated plan, taking into consideration the actual configuration/orientation of the arrays during each orbit; (5) displaying of eclipse and isolation timelines; and (6) displaying the "time to hazard" for longeron shadowing, as line graphs on timelines.

SACE is a fully delivered capability enhancing the flight control real-time monitoring of the ISS solar arrays and associated constraints management. Development continues to certify the flight planning capability for solar array operations.

\section{H. Power Management}

The ISS flight control team currently utilizes a complex suite of tools to plan and execute management of the ISS power systems. The Power Planning and Analysis Tool (PLATO) project is a collaboration project intended to consolidate all the ISS power planning tasks into a single application with an easily manipulated interface, a front end appearance similar to OSTPV, and which will automate the sharing of power-related data with other flight control disciplines. ${ }^{11}$ It would still maintain the robust capability of the current power analysis toolset, allowing the qualified user to delve into the underlying interface and modify the analysis parameters just as ISS flight control team can today with the MOD Integrated Planning System (IPS) tools. Ames is providing the technology infusion to this tool development while the MOD team is providing much of the user interface and integration development. As with the SACE project, this project leverages off the Ames EUROPA engine, which provides constraint management and reasoning, decision-support and planning.

\section{Constraints and Flight Rules Management}

In preparing for mission operations, the MOD flight control team must understand, document, utilize, and account for many operational constraints. A complicated variety of tools and processes are employed today in dealing with these flight constraints, and the Constraints and Flight Rules Management (ConFRM) was envisioned as a tool to manage these Ops constraints. ${ }^{12}$ ConFRM would provide a database-like storage of ops-related constraints, such as planning constraints, Flight Rules, and flight controller workstation limits, associated with each mission. ConFRM would provide a standardized authoring tool for Flight Rules, and Ground Rules and Constraints with the ability to export into desired formats. It would have the ability to link all related constraints/products, would capture full history/heritage of a constraint (improves Knowledge Management), have the ability to import/export constraint data to/from other MOD Tools (NGPS, MCT, etc.), and have the ability to identify mismatches between constraints in various input/output products, providing constraint product Quality Assurance. This project is currently on hold pending budget resolution.

\section{J. Attitude Planning}

The ISS Attitude Determination and Control Officer (ADCO) has overall responsibility for the integration of all Guidance, Navigation and Control, propulsion and Interim Control Module activities (including monitoring CMGs). ADCO works in partnership with Russian controllers to manage the station's orientation, controlled by the onboard Motion Control Systems. This position also plans and calculates future orientations and maneuvers for the station. The ADCO Planning Exchange Tool (APEX) will streamline the existing manual and time-intensive ADCO attitude planning tools into a more automated, user-friendly application that interfaces with existing products and allows the ADCO to produce accurate products and timelines more efficiently.

\section{Autonomous Mission Operations}

The Autonomous Mission Operations (AMO) project within the Advanced Exploration Systems (AES) will assess how to operate a crewed mission with a long communication delay between the spacecraft and Earth. The Project lead is at ARC, and it is supported by JSC, MSFC, KSC, and LaRC. For the last 50 years, NASA has operated human space missions primarily from Earth. This mode of operations has several advantages: by having a large part of the people involved on the ground the on board crew could be smaller, the vehicles could be simpler

and lighter, and the mission performed for a lower cost. However, as we explore beyond the Moon, that will no longer be possible. Future human spaceflight missions will place crews at large distances and light-time delays from 
Earth. The one-way light-time delay to the Moon is 1.2 seconds, which is sufficient to make continuous control (e.g. for landing) difficult or impossible to conduct from Earth. One-way light time delays to destinations such as Near Earth Asteroids (NEAs) range from seconds to minutes, at perigee. The one-way light-time delay to Mars ranges from 3 minutes (at conjunction) to 22 minutes (at opposition).

The wide variety of mission operations activities performed by ground today assume zero light time delay communication between ground and crew. Even today, critical events and failures require either automatic systems onboard the spacecraft or the crew to respond and safe the vehicle. For example, complex events like rendezvous and proximity operations are completed with assistance from Mission Control. As light time delays increase, the communications delay between the ground and the spacecraft will grow greater. The number of situations in which crew autonomy or spacecraft automation are required to conduct the mission is expected to increase. By contrast, the flight control team will transition to a role more involved with data analysis, system prognostics and near-real time support and less with immediate oversight of the flight crew's activities.

There are significant open questions regarding which mission operations functions to allocate to ground, and which to allocate to crew, as the time delay increases:

- How should mission operations responsibilities be allocated between ground and the spacecraft in the presence of significant light-time delay between the spacecraft and the Earth?

- How should ground-based planning, monitoring, and control be distributed across the flight control team and ground system automation?

- How should spacecraft-based planning, monitoring, and control be distributed between the flight crew and onboard system automation?

- When during the mission should responsibility shift from flight control team to crew or from crew to vehicle, and what should the process of shifting responsibility be as the mission progresses?

- What tools and technologies are needed to support these missions, and what are their costs?

NASA's Autonomous Mission Operations project will perform a series of experiments, using advanced mission operations technology, in order to answer these questions. The experiments will evaluate the performance of a flight control team and spacecraft crew in an analog environment. The team will conduct a series of mission activities with varying operational scenarios, differing levels of crew autonomy, and varying time delays. The experimental design will reveal how well crew autonomy enables the joint team to perform the mission.

\section{Conclusion}

NASA's Ames Research Center and Johnson Space Center are working together to apply intelligent systems to mission operation tools and systems. This work is critical to the Agency, ISS and any future human exploration programs. Improving the capacity of NASA's main manned mission operations teams to handle more operations per controller, enhancing the capabilities of those teams to handle complex decisions in a timelier manner, and increasing the available knowledge to the flight controller to make safer decisions are the critical motivations for this work.

This paper reports the latest projects in a planned series of efforts to greatly enhance how Mission Operations are performed for human spaceflight within NASA. These efforts will enhance the current mission operations of the International Space Station and prepare MOD for the more automated and autonomous exploration spacecraft in the future human exploration missions. This paper also reports the Automation Mission Operations assessments associated with preparing the agency for future exploration missions related to operations support. 


\section{Appendix A}

\section{Acronym List}

\begin{tabular}{|c|c|}
\hline AES & Advanced Exploration Systems \\
\hline ADCO & Attitude Determination and Control Officer \\
\hline AMISS & Anomaly Monitoring Inductive Software System \\
\hline AMO & Autonomous Mission Operations \\
\hline APEX & ADCO Planning Exchange Tool \\
\hline ARC & Ames Research Center \\
\hline ATV & Autonomous Transfer Vehicle \\
\hline BRAHMS & Business Redesign Agent-based Holistic Modeling System \\
\hline CMG & Control Moment Gyroscope \\
\hline ConFRM & Constraints and Flight Rules Management \\
\hline CPS & Consolidated Planning System \\
\hline CSA & Canadian Space Agency \\
\hline ESA & European Space Agency \\
\hline ETCS & External Thermal Control System \\
\hline EUROPA & Extensible Universal Remote Operations Planning Architecture \\
\hline HEFT & Human Exploration Framework Team \\
\hline HTV & H-II Transfer Vehicle \\
\hline IMS & Inductive Monitoring System \\
\hline IPS & Integrated Planning System \\
\hline ISS & International Space Station \\
\hline JEM & Japanese Experiment Module \\
\hline JSC & Johnson Space Center \\
\hline KSC & Kennedy Space Center \\
\hline LaRC & Langley Research Center \\
\hline $\mathrm{MCC}$ & Mission Control Center \\
\hline MCC-M & Mission Control Center in Moscow \\
\hline MCT & Mission Control Technologies \\
\hline MOD & Mission Operations Directorate \\
\hline MSFC & Marshal Space Flight Center \\
\hline MSL & Mars Science Laboratory \\
\hline NASA & National Aeronautics and Space Administration \\
\hline NEOs & Near-Earth Objects \\
\hline NGPS & Next Generation Planning System \\
\hline OCA & Orbital Communication Adapter \\
\hline OCAMS & Orbital Communication Adapter Mirroring System \\
\hline OPTimIS & Operations Planning Timeline Integration System \\
\hline OSTPV & On-Board Short Term Plan Viewer \\
\hline OTF & Operations Test Facility \\
\hline PLATO & Power Planning and Analysis Tool \\
\hline POIC & Payload Ops Integration Center \\
\hline SACE & Solar Array Constraint Engine \\
\hline SPIFe & Scheduling and Planning Interface for Exploration \\
\hline STAR & Scheduling, Training Administration, and Records \\
\hline STP & Short Term Plan \\
\hline TAMS & Training Administration Management System \\
\hline XSearch & Cross Search \\
\hline
\end{tabular}




\section{Acknowledgments}

The Authors wish to acknowledge all of the multiple project leads and team members at Ames, JSC, MSFC, $\mathrm{KSC}$, and LaRC for the above-mentioned projects. Their work is changing the way that mission operations is performed for the International Space Station and enabling NASA to be ready to fully support future human exploration programs.

\section{References}

${ }^{1}$ Mishkin, A., Lee, Y., Korth, D., and LeBlanc, T., "Integrated Human-Robotic Missions to the Moon and Mars: Mission Operations Design Implications," IEEEAC paper \#1400, Version 2, Updated November 2, 2006

${ }^{2}$ Muscettola, N., Nayak, P., Pell, B., and Williams, B., "Remote agent: To boldly go where no AI system has gone before," Artificial Intelligence, 103(1-2):5 - 48, 1998.

${ }^{3}$ Bresina, J., Jónsson, A., Morris, P., and Rajan, K., "MAPGEN: Mixed Initiative Planning and Scheduling for the Mars '03 MER Mission,” International Symposium on AI and Robotics in Space, Nara, Japan, 2003.

${ }^{4}$ Frank, J., Morris, P.H., Greene, J., Hall, T., "The Challenge of Evolving Mission Operations Tools for Manned Spaceflight," 9th International Symposium on Artificial Intelligence, Robotics, and Automation for Space, Los Angeles, CA, 2008; Clement, B., Barreiro, J., "Spatial Planning for International Space Station Crew Operations," Proceedings of the International Symposium on Artificial Intelligence, Robotics and Automation in Space, 2010.

${ }^{5}$ Sierhuis, M., Clancey, W. J., van Hoof, R. J., Seah, C. H., Scott, M. S., Nado, R. A., Blumenberg, S. F., Shafto, M. G., Anderson, B. L., Bruins, A. C., Buckley, C. B., Diegelman, T. E., Hall, T. A., Hood, D., Reynolds, F. F., Toschlog, J. R. and Tucker, T., "NASA's OCA Mirroring System: An application of multiagent systems in Mission Control," in S. Decker, Sierra, and Castelfranchi (ed.) 8th Int. Conf. on Autonomous Agents and Multiagent Systems (AAMAS 2009). Budapest, Hungary: International Foundation for Autonomous Agents and Multi-agent Systems (www.ifaamas.org).

${ }^{6}$ Clancey, W. J., Sierhuis, M., Seah, C., Buckley, C., Reynolds, F., Hall, T., Scott, M. "Multi-agent simulation to implementation: A practical engineering methodology for designing space flight operations." In A. Artikis, G. O'Hare, K. Stathis, \& G. Vouros (Eds.), Engineering Societies in the Agents' World VIII. Lecture Notes in Computer Science Series, Volume 4870. Heidelberg Germany: Springer, 2008, pp. 108-123.

${ }^{7}$ Iverson, D. L., "Data Mining Applications for Space Mission Operations System Health Monitoring", Proceedings of the SpaceOps 2008 Conference, ESA, EUMETSAT, AIAA, Heidelberg, Germany, May 2008

${ }^{8}$ Trimble, J., Crocker, A., "A Flexible Evolvable Architecture for Constellation Mission Systems User Applications," SpaceOps 2008, Heidelberg Germany, May 12-16, 2008

${ }^{9}$ Keller, R.M., Wolfe, S.R., Windrem, M.N., and Berrios, D.C., "XSearch: A System for Searching and Interrelating NASA Mission Operations Data," SpaceOps 2008 Conference, Heidelberg, Germany, May 2008.

${ }^{10}$ Reddy, S.Y., Ai Chang, M., Iatauro, M., Kurklu, E., Boyce, M., Frank, J., Jonsson, A., "Planning and Monitoring Solar Array Operations on the International Space Station," Proceedings of the Workshop on Planning and Scheduling Applications, in conjunction with the International Conference on Automated Planning, 2008

${ }^{11}$ Barreiro, J., Jones, G., Schaffer, J., "Peer-to-peer Planning for Space Mission Control," Proceedings of the 6th International Workshop on Planning and Scheduling for Space, (IWPSS) Pasadena, CA., 2009; Aghevli, A., Bachmann, A., Bresina, J.L., Greene, J., Kanefsky, R., Kurien, J.,McCurdy, M., Morris, P.H., Pyrzak, G., Ratterman, C., Vera, A., Wragg. S., "Planning Applications for Three Mars Missions with Ensemble," 5th International Workshop on Planning and Scheduling for Space. (IWPSS) Baltimore, MD, 2007

${ }^{12}$ Barreiro, J., Chachere, J., Frank, J., Bertels, C., Crocker, A., "Constraint and Flight Rule Management for Space Mission Operations," Proceedings of the International Symposium on Artificial Intelligence, Robotics and Automation in Space, 2010. 